\title{
A new technique to determine fusion barrier heights using proximity poten- tials
}

\author{
Raj Kumari ${ }^{1, a}$ and Aditi Toshniwal ${ }^{2}$ \\ ${ }^{1}$ Department of Physics, Panjab University, Chandigarh -160 014, India \\ ${ }^{2}$ S. V. National Institute of Technology, Surat - 395007, India
}

\begin{abstract}
We develop a new technique to calculate fusion barrier heights of any fixed target reaction series. We calculate the barrier heights for the fusion reactions of ${ }^{119} \mathrm{Sn}$ and ${ }^{197} \mathrm{Au}$ targets using this technique. This technique is simple and very useful for estimating the fusion barrier heights for those reactions for which empirical values are not available. A formula is derived by performing theoretical calculations using different versions of proximity potential. Using this formula, we can predict the barrier height for the fusion reaction of any projectile with ${ }^{119} \mathrm{Sn}$ or ${ }^{197} \mathrm{Au}$ target using the empirical barrier height for the fusion reaction of same projectile with ${ }^{62} \mathrm{Ni}$ target. In order to check the accuracy of this technique, the fusion parameters calculated by this new technique are compared with the fusion parameters calculated by using parameterized formulae presented in a systematic study conducted by using double folding model.
\end{abstract}

\section{Introduction}

Our understanding of the fusion reactions between the strongly-bound as well as weakly-bound nuclei have been enriched due to the continuous efforts devoted by nuclear community in the field of theory [1-10] and experiments [11-15]. The knowledge of the mechanism of heavyion fusion reaction is very crucial to explore the nucleusnucleus potential as well as for the synthesis of super heavy elements. At low incident energies, i.e. $(\mathrm{E}<10$ $\mathrm{MeV} /$ nucleon), phenomena like fusion, fission, elastic and inelastic scattering cannot be studied without the knowledge of nucleus-nucleus potential. Even at intermediate energies, an accurate knowledge of the total interaction potential is necessary to study various observables like multifragmentation [16-18], collective flow [19-21] etc.

It is well known that the threshold energy required for the fusion to take place cannot be given by the Coulomb barrier alone. The nuclear potential is equally important in determining the fusion barrier. The Coulomb part of interaction potential is well known. In order to calculate the total interaction potential $V_{T}(R)=V_{N}(R)+V_{C}(R)$, the precise knowledge of the nuclear potential $V_{N}(R)$ is required.

Many attempts have been put forward to give simple and accurate form of nuclear potential. Among such attempts, proximity model [22-25] is well known for its simplicity. In this model, the nuclear part of the interaction potential is given as the product of the geometrical factor and the universal function. The universal function is function of separation between the surfaces of two colliding nuclei (s) and therefore, it is independent of the masses of

\footnotetext{
a e-mail: rajkumari80pu@gmail.com
}

the colliding nuclei. The separation between the two colliding nuclei is related to the internuclear distance between the centers of the colliding nuclei through their nuclear radii. So we need accurate knowledge of nuclear radii.

In the present study, we propose a new technique, which can be used to calculate the fusion barrier heights of any fixed target reaction series. For this study, we first compute barrier heights for $\mathrm{X}+{ }^{62} \mathrm{Ni}, \mathrm{X}+{ }^{119} \mathrm{Sn}$ and $\mathrm{X}+$ ${ }^{197} \mathrm{Au}$ reaction series using different versions of proximity potential. Here, $\mathrm{X}$ projectile varies from ${ }^{1} \mathrm{H}$ to ${ }^{59} \mathrm{Co}$. This study is carried out using proximity potentials PROX 77, PROX 88, PROX 2000, PROX 2000DP, PROX 2010 and MOD-PROX 88.

\section{Description of Models}

The proximity potential is based on the theorem which states that "the force between two gently curved surfaces in close proximity is proportional to the interaction potential per unit area between two flat surfaces" [22].

\subsection{Proximity 1977 (PROX 77)}

The interaction potential $V_{N}(R)$ between two colliding surfaces is given by

$$
V_{N}=4 \pi \gamma b \bar{C} \Phi\left(\frac{R-C_{1}-C_{2}}{b}\right) \mathrm{MeV}
$$

where $\bar{C}\left(=\left(C_{1} C_{2}\right) /\left(C_{1}+C_{2}\right)\right), b$ and $R$ are the reduced radius, surface width and central separation, respectively. Here, $C_{i}$ is the central radius given by

$$
C_{i}=R_{i}\left[1-\left(\frac{b}{R_{i}}\right)^{2}+\ldots . .\right]
$$


and $\Phi$ is the universal function. The effective sharp radius $R_{i}$ is written as

$$
R_{i}=1.28 A_{i}^{1 / 3}-0.76+0.8 A_{i}^{-1 / 3} \mathrm{fm}(i=1,2) .
$$

The separation between the half-density surfaces of two colliding nuclei is given by

$$
s=R-C_{1}-C_{2} .
$$

The surface energy coefficient $\gamma$ has the form

$$
\gamma=\gamma_{0}\left[1-k_{s}\left(\frac{N-Z}{A}\right)^{2}\right] .
$$

Here $\gamma_{0}$ and $k_{s}$ are the surface energy coefficient and surface asymmetry constant, respectively. Here, $\mathrm{N}$ and $\mathrm{Z}$ are the total number of neutrons and protons respectively. The values of $\gamma_{0}$ and $k_{s}$ are taken to be $0.9517 \mathrm{MeV} / \mathrm{fm}^{2}$ and 1.7826 respectively.

\subsection{Proximity 1988 (PROX 88)}

The values of coefficients $\gamma_{0}$ and $k_{s}$ were modified by Moller and Nix [26] for more refined mass formula and provide the values $1.2496 \mathrm{MeV} / \mathrm{fm}^{2}$ and 2.3 respectively.

\subsection{Proximity 2000 (PROX 2000)}

Using droplet model concept for the surface tension coefficients and with advance understanding in nuclear radii, Myers and Światecki [27] included the neutron skin of a nucleus $\left(t_{i}\right)$ as well as half-density radii of the charge distribution $\left(c_{i}\right)$ in the calculations of matter radius $\left(C_{i}\right)$. The contribution of neutron skin was also taken in the calculations of surface energy coefficient $(\gamma)$. This potential was proposed to remove inconsistency of $4 \%$ between the results of PROX 77 and experimental data.

\subsection{Proximity 2000 DP (PROX 2000DP)}

I. Dutt and R. K. Puri in Ref. [28] modified PROX 2000 by including the nuclear radii given by Royer and Rousseau [29]. This modified version is named as PROX 2000DP.

\subsection{Proximity 2010 (PROX 2010)}

This version [30] was introduced by replacing the form of nuclear charge radius in Prox 2000 with the form given by Royer and Rousseau [29]. The values of surface energy constant $\gamma_{0}$ and surface asymmetry constant $k_{s}$ were taken to be $1.25284 \mathrm{MeV} / \mathrm{fm}^{2}$ and 2.345 respectively. The form of universal function $\Phi(\xi)$ was taken from Blocki et al. [31].

\subsection{Modified Proximity 1988 (MOD-PROX 88)}

The value of surface energy constant $\gamma_{0}$ used in PROX 88 is slightly adjusted from $1.2496 \mathrm{MeV} / \mathrm{fm}^{2}$ to 1.65 $\mathrm{MeV} / \mathrm{fm}^{2}$ by fitting the fusion cross section of various reactions simultaneously within the extended Wong Model.

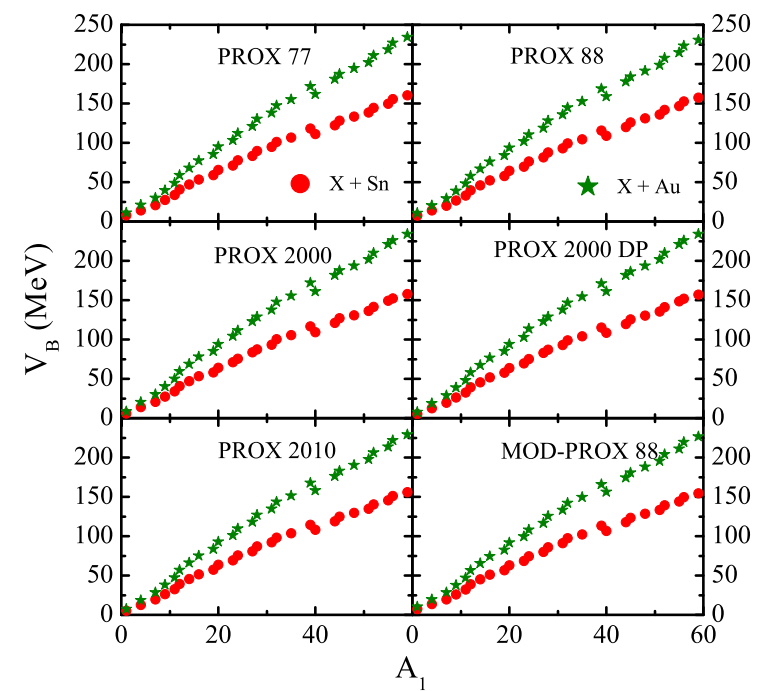

Figure 1. (Color online) The barrier heights $V_{B}(\mathrm{MeV})$ are displayed as a function of mass of the projectile $\left(A_{1}\right)$. The circles (red) and stars (green) represent reactions of projectile $\mathrm{X}$ with target ${ }^{119} \mathrm{Sn}$ and target ${ }^{197} \mathrm{Au}$ respectively. Here, $\mathrm{X}$ projectile varies from ${ }^{1} \mathrm{H}$ to ${ }^{59} \mathrm{Co}$.

This modified version [32] has a strong attraction as compared to the other versions of proximity potential.

Using all above mentioned models, the nuclear part of the total interaction potential is computed. The total interaction potential is calculated by adding the Coulomb potential to the nuclear part.

Once the total interaction potential is known, the fusion barrier heights and positions are calculated using the following relations:

$$
\left.\frac{d V(R)}{d R}\right|_{R=R_{B}}=0,\left.\quad \frac{d^{2} V(R)}{d R^{2}}\right|_{R=R_{B}} \leq 0 .
$$

\section{Calculations and Discussions}

\subsection{Theoretical Study using proximity potentials}

In the present study, we first calculate the fusion barrier heights for three different reaction series (i.e. $\mathrm{X}+{ }^{62} \mathrm{Ni}$, $\mathrm{X}+{ }^{119} \mathrm{Sn}$ and $\mathrm{X}+{ }^{197} \mathrm{Au}$ ) using the above mentioned versions of proximity potential. Here, $\mathrm{X}$ projectile varies from ${ }^{1} \mathrm{H}$ to ${ }^{59} \mathrm{Co}$.

From Fig. 1, we can see that barrier heights increase monotonically with the mass of the projectile for fixed target reactions. This rise is steeper in case of Au target as compared to Sn target.

In Fig. 2, the percentage difference $\Delta V_{B} \%$ is displayed as a function of mass of the projectile $\left(A_{1}\right)$. The percentage difference $\Delta V_{B} \%$ for $\mathrm{X}+{ }^{119} \mathrm{Sn}$ and $\mathrm{X}+{ }^{197} \mathrm{Au}$ reaction series are calculated by using the relations

$$
\Delta V_{B} \%\left(X+{ }^{119} S n\right)=\frac{V_{B}\left(X+{ }^{119} S n\right)}{V_{B}\left(X+{ }^{62} N i\right) \times Z\left({ }^{119} S n\right)} \times 100,
$$




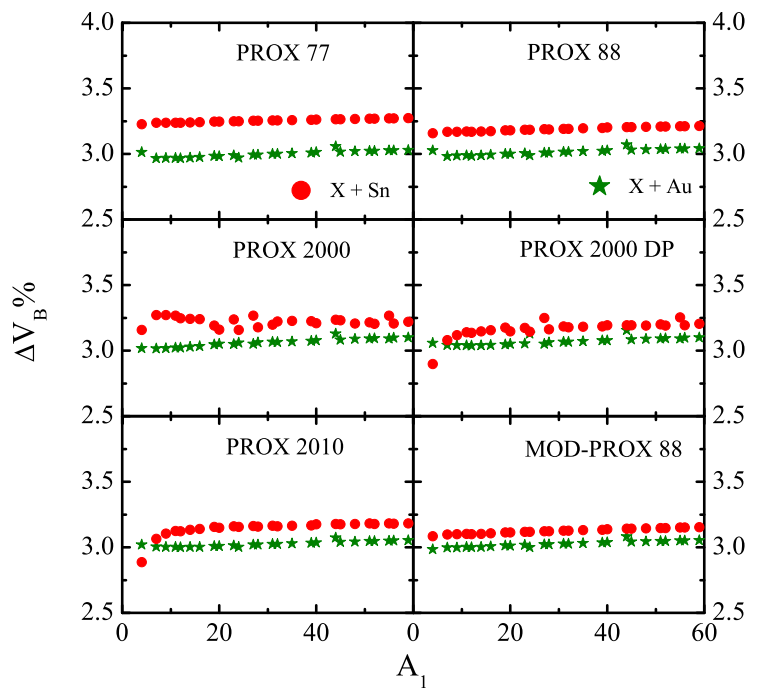

Figure 2. (Color online) The percentage barrier heights $\left(\Delta V_{B} \%\right)$ are displayed as a function of mass of the projectile. Symbols have same meaning as in Fig. 1.

and

$$
\Delta V_{B} \%\left(X+{ }^{197} A u\right)=\frac{V_{B}\left(X+{ }^{197} A u\right)}{V_{B}\left(X+{ }^{62} N i\right) \times Z\left({ }^{197} A u\right)} \times 100,
$$

respectively.

We find that $\Delta V_{B} \%$ for $\mathrm{X}+{ }^{119} \mathrm{Sn}$ and $\mathrm{X}+{ }^{197} \mathrm{Au}$ reaction series calculated using barrier heights for $\mathrm{X}+{ }^{62} \mathrm{Ni}$ reaction series and scaling w.r.t charge of fixed target comes out to be constant i.e. independent of the mass of the projectile (see Fig. 2). This constant factor comes out to be approximately 3.25 and 3.0 in cases of ${ }^{119} \mathrm{Sn}$ and ${ }^{197} \mathrm{Au}$ targets, respectively. This is due to the reason that since Coulomb potential is playing a major role so upon scaling w.r.t to the charge of the fixed target, the effect of Coulomb is reduced. The constant percentage difference represents that the mass of the projectile in these reactions is not playing significant role in the fusion process.

\subsection{Derivation}

In particular, the fusion barrier heights for $\mathrm{X}+{ }^{119} \mathrm{Sn}$ and $\mathrm{X}+{ }^{197} \mathrm{Au}$ reactions can be calculated using the relations

$$
V_{B}\left(X+{ }^{119} S n\right)=\frac{3.25 \times V_{B}\left(X+{ }^{62} N i\right) \times Z\left({ }^{119} S n\right)}{100}
$$

and

$$
V_{B}\left(X+{ }^{197} A u\right)=\frac{3.00 \times V_{B}\left(X+{ }^{62} N i\right) \times Z\left({ }^{197} A u\right)}{100} .
$$

This technique is named as scaling formula. Using this technique, we can calculate the barrier height for the reaction of any projectile $\mathrm{X}$ with ${ }^{119} \mathrm{Sn}$ or ${ }^{197} \mathrm{Au}$ using empirical barrier height of corresponding $\mathrm{X}+{ }^{62} \mathrm{Ni}$ reaction.
Table 1. Fusion barrier heights $V_{B}$ (empirical $\left./ D F^{P a r}\right)(\mathrm{MeV})$ for $\mathrm{X}+{ }^{62} \mathrm{Ni}$ reactions.

\begin{tabular}{|c|c|c|c|}
\hline Reaction & Empirical & Ref. & DF $^{\text {Par }}[33]$ \\
\hline \hline${ }^{16} \mathrm{O}+{ }^{62} \mathrm{Ni}$ & 31.12 & {$[34]$} & 30.14 \\
${ }^{30} \mathrm{Si}+{ }^{62} \mathrm{Ni}$ & 52.20 & {$[35]$} & 50.36 \\
${ }^{35} \mathrm{Cl}+{ }^{62} \mathrm{Ni}$ & 60.80 & {$[36]$} & 59.67 \\
${ }^{37} \mathrm{Cl}+{ }^{62} \mathrm{Ni}$ & 60.27 & {$[37]$} & 59.56 \\
${ }^{40} \mathrm{Ca}+{ }^{62} \mathrm{Ni}$ & 70.70 & {$[38]$} & 69.42 \\
\hline
\end{tabular}

\subsection{Calculations}

Table 2. The fusion barrier heights $V_{B}(\mathrm{MeV})$ calculated by using our new technique (scaling formula) and $D F^{\text {Par }}$ (for comparison) are presented.

\begin{tabular}{|l|c|c|}
\hline Reaction & Scaling formula & $\mathrm{DF}^{\text {Par }}[33]$ \\
\hline \hline${ }^{16} \mathrm{O}+{ }^{119} \mathrm{Sn}$ & 50.57 & 49.45 \\
${ }^{30} \mathrm{Si}+{ }^{119} \mathrm{Sn}$ & 84.83 & 82.88 \\
${ }^{35} \mathrm{Cl}+{ }^{119} \mathrm{Sn}$ & 98.80 & 98.47 \\
${ }^{37} \mathrm{Cl}+{ }^{119} \mathrm{Sn}$ & 97.94 & 98.30 \\
${ }^{40} \mathrm{Ca}+{ }^{119} \mathrm{Sn}$ & 114.89 & 114.66 \\
${ }^{16} \mathrm{O}+{ }^{197} \mathrm{Au}$ & 73.75 & 72.34 \\
${ }^{30} \mathrm{Si}+{ }^{197} \mathrm{Au}$ & 123.71 & 121.63 \\
${ }^{35} \mathrm{Cl}+{ }^{197} \mathrm{Au}$ & 144.01 & 144.74 \\
${ }^{37} \mathrm{Cl}+{ }^{197} \mathrm{Au}$ & 142.84 & 144.50 \\
${ }^{40} \mathrm{Ca}+{ }^{197} \mathrm{Au}$ & 167.56 & 168.66 \\
\hline
\end{tabular}

In Table 1, empirical barrier heights for few reactions with ${ }^{62} \mathrm{Ni}$ target are presented along with barrier heights calculated using $D F^{P a r}$ (parameterized formulae using double folding model) [33]. Here we can see that the parameterized double folding calculations are in close agreement with the empirical values. Therefore, we can use these parameterized formulae for comparing our results using this new technique. Using the empirical values (in table 1), the fusion barrier heights for the reactions of same projectiles but with ${ }^{119} \mathrm{Sn}$ target and ${ }^{197} \mathrm{Au}$ target are calculated using scaling formula (Eqs. 9 and 10) and these values are displayed in Table 2. Since the empirical barrier heights for all these systems (in table 2) are not available yet, so the calculated values are compared with the values calculated using parameterized formulae derived from double folding model. A close agreement between the two values suggests that this new technique is quite accurate and simple and therefore, can be used to calculate barrier heights for all those reactions for which empirical values are not available.

\section{Summary}

Using different versions of proximity potentials, we developed a technique (scaling formula) to calculate fusion barrier heights of any fixed target reaction series. In particular, we calculated the barrier heights for the fusion reactions of few projectiles with ${ }^{119} \mathrm{Sn}$ and ${ }^{197} \mathrm{Au}$ targets using the barrier heights for the reactions of same projectiles with ${ }^{62} \mathrm{Ni}$ target. This new formula can be used to find barrier heights of any fixed target/fixed beam reaction series 
for which empirical value is not available and hence can be useful in the synthesis of super-heavy elements.

\section{Acknowledgment}

One of the authors R. K. acknowledges the financial supports from Council of Scientific and Industrial Research (CSIR), New Delhi. Authors acknowledge collaboration with Professor Rajeev K. Puri.

\section{References}

[1] R. K. Puri, R. Arora and R. K. Gupta, Phys. Rev. C 60, 054619 (1999).

[2] R. Kumari, Nucl. Phys. A 917, 85 (2013).

[3] C. Simenel, Eur. Phys. J. A 48, 152 (2012).

[4] L. C. Chamon et al., Phys. Rev. C 66, 014610 (2002).

[5] C. H. Dasso, S. Landowne and A. Winther, Nucl. Phys. A 432, 495 (1985).

[6] C. Simenel, M. Dasgupta, D. J. Hinde and E. Williams, Phys. Rev. C 88, 064604 (2013).

[7] A. S. Umar, C. Simenel and V. E. Oberacker, Phys. Rev. C 89, 034611 (2014).

[8] C. Simenel and B. Avez, Int. J. Mod. Phys. E 17, 31 (2008).

[9] A. B. Balantekin and N. Takigawa, Rev. Mod. Phys. 70, 77 (1998).

[10] H. Esbensen and S. Landowne, Phys. Rev. C 35, 2090 (1987).

[11] J. J. Kolata et al., Phys. Rev. C 85, 054603 (2012).

[12] A. Pakou et al., Phys. Rev. C 87, 0146193 (2013).

[13] G. Montagnoli et al., Phys. Rev. C 85, 024607 (2012).

[14] J. R. Leigh et al., Phys. Rev. C 52, 3151 (1995).

[15] C. R. Morton et al., Phys. Rev. Lett. 72, 4074 (1994).
[16] Y. K. Vermani et al., J. Phys. G: Nucl. Part. Phys. 37, 015105 (2010).

[17] S. Goyal and R. K. Puri, Phys. Rev. C 83, 047601 (2011).

[18] S. Kaur and R. K. Puri, Phys. Rev. C 87, 014620 (2013).

[19] S. Gautam et al., Phys. Rev. C 86, 034607 (2012).

[20] R. Bansal et al., Phys. Rev. C 87, 01601 (2013).

[21] R. Bansal et al., J. Phys. G: Nucl. Phys. 41, 035103 (2014).

[22] J. Blocki, J. Randrup, W. J. Światecki, and C. F. Tsang, Ann. Phys. 105, 427 (1977).

[23] I. Dutt and R. K. Puri, Phys. Rev. C 81, 064609 (2010).

[24] I. Dutt and R. K. Puri, Phys. Rev. C 81, 047601 (2010).

[25] I. Dutt and R. K. Puri, Phys. Rev. C 81, 064608 (2010).

[26] P. Moller and J. R. Nix, Nucl. Phys. A 361, 117 (1981).

[27] W. D. Myers and W. J. Światecki, Phys. Rev. C 62, 044610 (2000).

[28] I. Dutt and R. K. Puri, Phys. Rev. C 81, 044615 (2010).

[29] G. Royer and R. Rousseau, Eur. Phys. J. A 42, 541 (2009).

[30] I. Dutt and R. Bansal, Chin. Phys. Lett. 27, 112402 (2010).

[31] J. Blocki and W. J. Światecki, Ann. Phys (N.Y.) 132, 53 (1981).

[32] R. Kumar, Phys. Rev. C 84, 044613 (2011).

[33] W. W. Qu, G. L. Zhang and X. Y. Le, Nucl. Phys. A 868, 1 (2011) and references therein.

[34] J. O. Newton et al., Phys. Rev. C 70, 024605 (2004).

[35] A. M. Stefanini et al., Nucl. Phys. A 456, 509 (1986).

[36] W. Scobel et al., Phys. Rev. C 14, 1808 (1976).

[37] J. J. Vega et al., Phys. Rev. C 42, 947 (1990).

[38] B. Sikora et al., Phys. Rev. C 20, 2219 (1979). 